\title{
Long-Term Trends in Ischemic Stroke Incidence and Risk Factors: Perspectives from an Asian Stroke Registry
}

\author{
Benjamin Y.Q. Tan, ${ }^{\mathrm{a}, \mathrm{b}}$ Joshua T.C. Tan ${ }^{\mathrm{a}}$, Dawn Cheah, ${ }^{\mathrm{b}}$ Huili Zheng, ${ }^{\mathrm{c}}$ Pin Pin Pek, ${ }^{\mathrm{d}}$ Deidre A. De Silva, \\ Aftab Ahmad, ${ }^{\mathrm{f}}$ Bernard P.L. Chan, ${ }^{\mathrm{a}}$ Hui Meng Chang, ${ }^{\mathrm{e}}$ Keng He Kong, ${ }^{\mathrm{g}}$ Sherry H. Young, \\ Kok Foo Tang, ${ }^{\mathrm{i}}$ Tian Ming Tu, ${ }^{\mathrm{g}}$ Leonard Leong-Litt Yeo, ${ }^{\mathrm{a}, \mathrm{b}}$ Narayanaswamy Venketasubramanian, ${ }^{\mathrm{j}}$ \\ Andrew F.W. Ho, ${ }^{\mathrm{d}, \mathrm{k}}$ Marcus Eng Hock Ong ${ }^{\mathrm{d}, \mathrm{l}}$

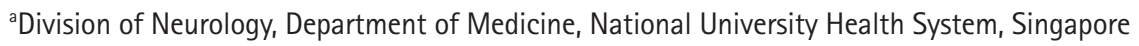 \\ ${ }^{b}$ Department of Medicine, Yong Loo Lin School of Medicine, National University of Singapore \\ 'National Registry of Diseases Office, Health Promotion Board, Singapore \\ ${ }^{d}$ Department of Emergency Medicine, Singapore General Hospital, Singapore \\ eDepartment of Neurology, National Neuroscience Institute, Singapore General Hospital Campus, Singapore \\ fDepartment of Neurology, Ng Teng Fong General Hospital, National University Health System, Singapore \\ ${ }^{9}$ Department of Neurology, National Neuroscience Institute, Tan Tock Seng Hospital, Singapore

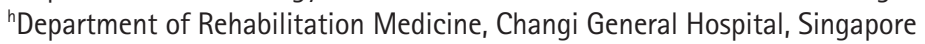 \\ Tang Neurology \& Medical Clinic, Mount Elizabeth Medical Centre, Singapore

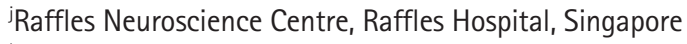 \\ ${ }^{k}$ Cardiovascular \& Metabolic Disorders Program Duke-National University of Singapore Medical School, Singapore \\ 'Health Services Research Centre, Singapore Health Services, Singapore
}

\section{Dear Sir:}

Risk factors of acute ischemic stroke (AIS) are well-recognised, ${ }^{1}$ but the relative importance of each contributing risk factor has evolved. Analysing the regional trends of these cardiovascular risk factors will enable the development of more targeted strategies to tackle AIS and reduce morbidity and disability. Few studies have evaluated inter-ethnic risk factor trends within a single co-localised population, ${ }^{2}$ especially amongst Asians, who have a different risk factor profile and consequently, stroke mechanisms. ${ }^{3}$ Singapore is uniquely placed to contribute to the understanding of these trends, given our multi-ethnic population (Supplementary Table 1).

The Singapore Stroke Registry (SSR) is an active nationwide prospective registry collecting data of stroke cases from all public hospitals in Singapore. ${ }^{4}$ Our study explored the interethnic trends of incidence and risk factors of AIS cases over a 12-year period using the SSR. These findings can be applied to the development of targeted strategies for the prevention of
AIS both locally and regionally.

A total of 60,325 AIS cases from 2005 to 2016 were analysed in 3-year quartiles with direct age standardisation using the 2005 to 2007 Singapore resident population as reference. Data was stratified into ethnic groups based on the Singapore Census classification: Chinese, Malay, Indian, or others: which comprises multiple different ethnic groups, a tiny proportion of the population, and thus were not analysed separately. Prevalence of risk factors amongst AIS cases was analysed within each time-period for each subgroup. Trends were analysed using Poisson, logistic, linear, or Cox regression, depending on the specific variables analysed.

There was a $11.6 \%$ decrease in the age-standardised incidence rate (ASIR) of AIS from 2005-2007 to 2014-2016 (Table 1). The decrease in ASIR was observed in both sexes (males 5.9\%; females 19.1\%). Stratifying by ethnic group, the ASIR had decreased in both Chinese (15.0\%) and Indians (11.2\%), but increased by $11.5 \%$ in Malays, and specifically, by $20.2 \%$ in Malay males. Amongst $\geq 65$-year-old cases, the crude incidence rate 


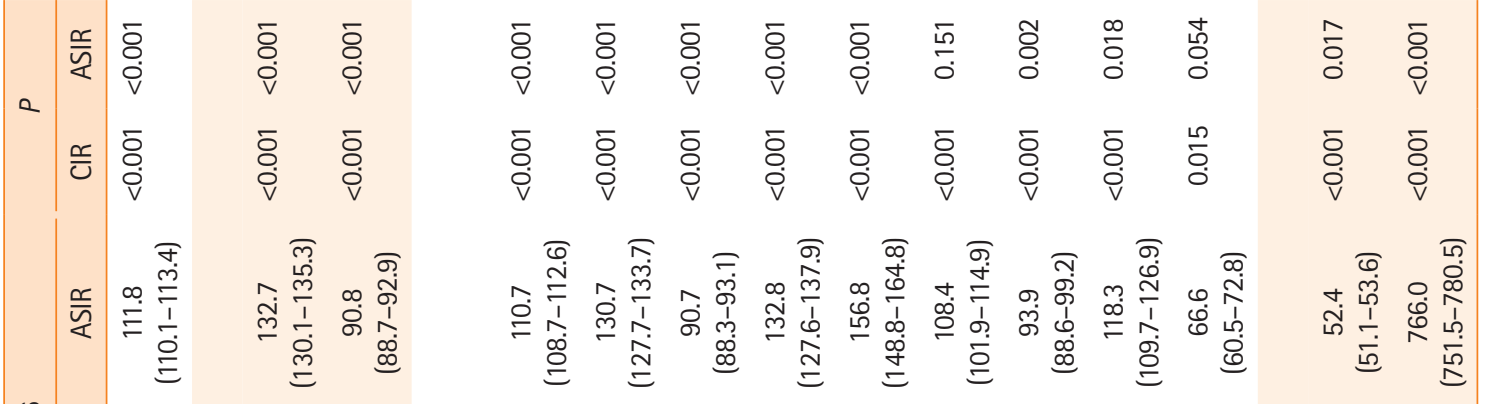

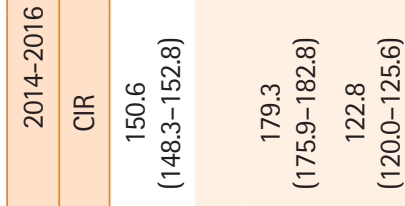

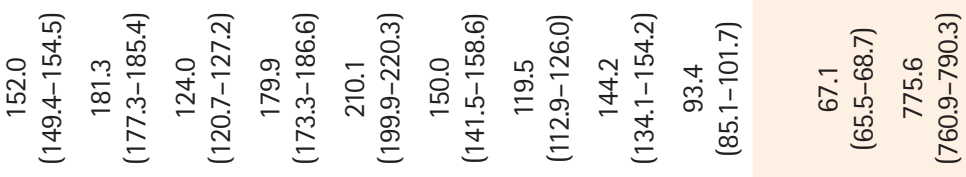

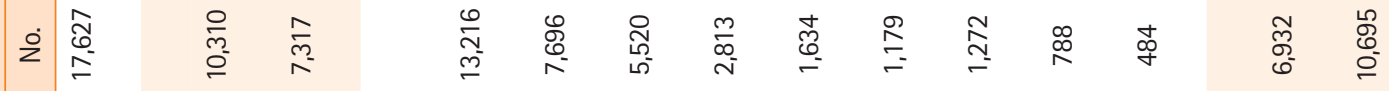

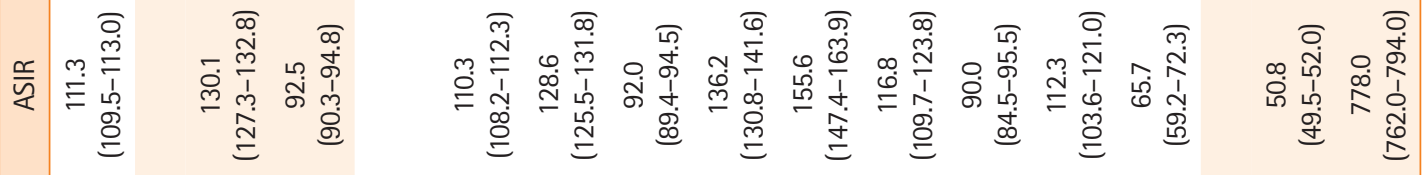

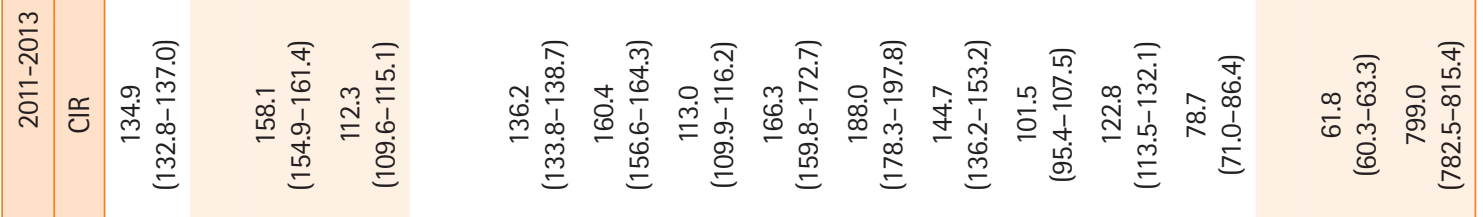

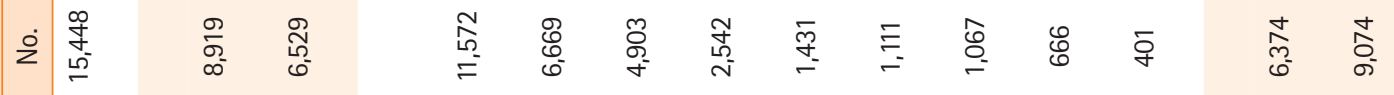

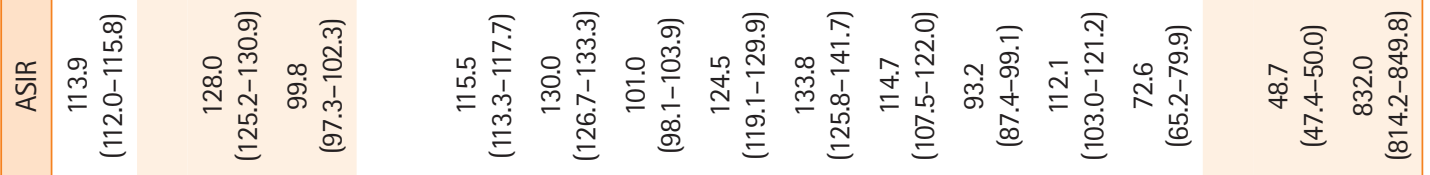

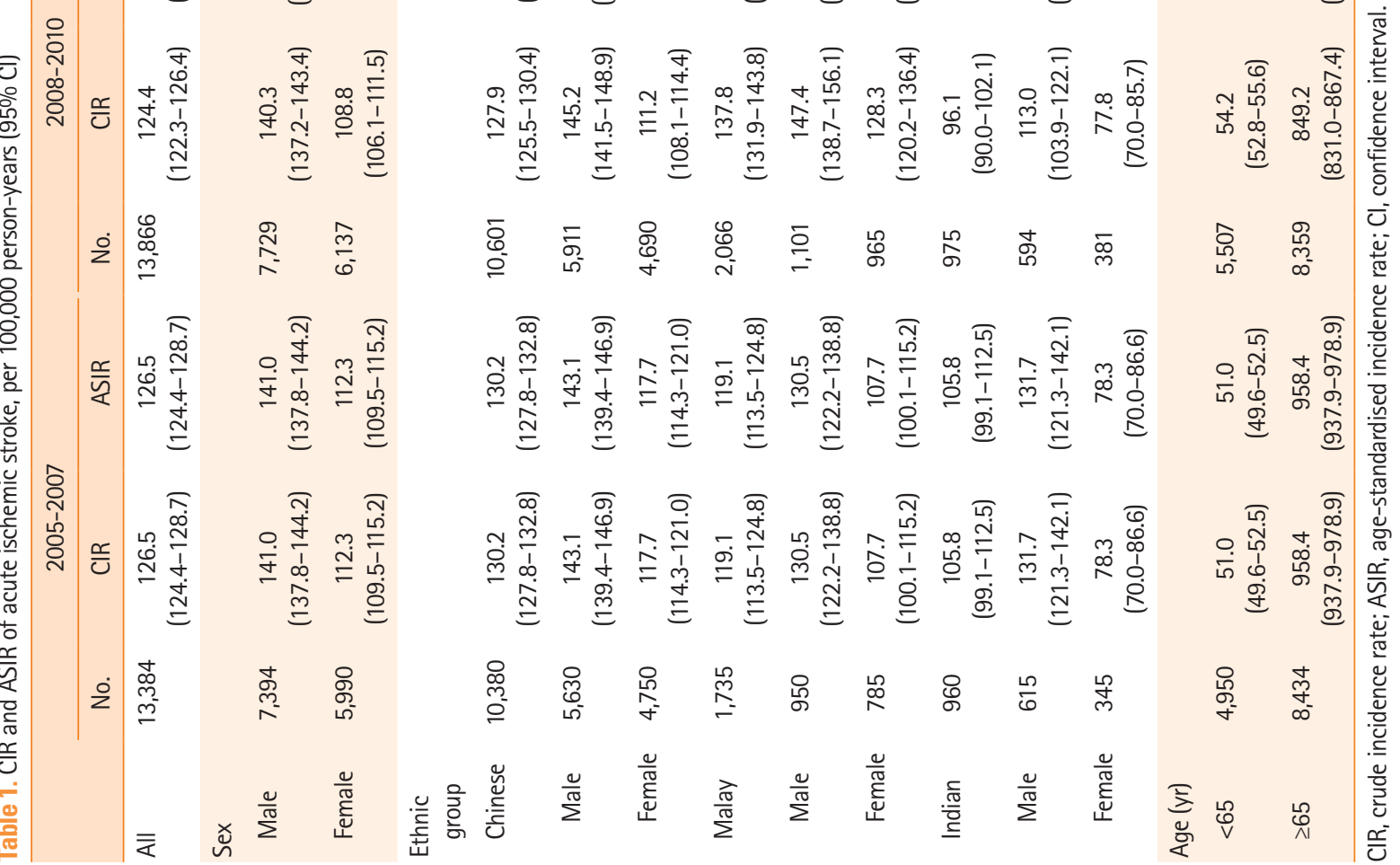


decreased by $19.1 \%$, as compared to $<65$-year-old cases, which saw a $31.6 \%$ increase.

Amongst AIS risk factors (Figure 1), history of stroke (6.3\% to $17.6 \%$ ), atrial fibrillation (AF; $15.8 \%$ to $25.2 \%$ ), hypertension $(80.2 \%$ to $100 \%)$, and hyperlipidaemia ( $79.9 \%$ to $100 \%)$ have increased in prevalence after adjusting for age, sex, and ethnic group. In contrast, the adjusted prevalence of smoking ( $41.2 \%$ to $34.6 \%$ ), and diabetes ( $46.2 \%$ to $43.2 \%$ ) have decreased. In terms of ethnic variation, AF was strikingly more prevalent in Chinese and Malays with AIS, in contrast to Indians. However, in all three ethnic groups, AF showed an increasing prevalence over time (Chinese: $16.5 \%$ to $24.2 \%, P<0.001$; Malays: $16.9 \%$ to $19.8 \%, P=0.011$; Indians: $7.1 \%$ to $12.3 \%$, $P=0.001)$. We also observed that AF was more prevalent in females (ranging between 19.8\% to 29.5\%) than males (between $12.6 \%$ to $17.8 \%$ ) across all time periods.

In subgroups that showed increased AIS incidence: $<65$-year-

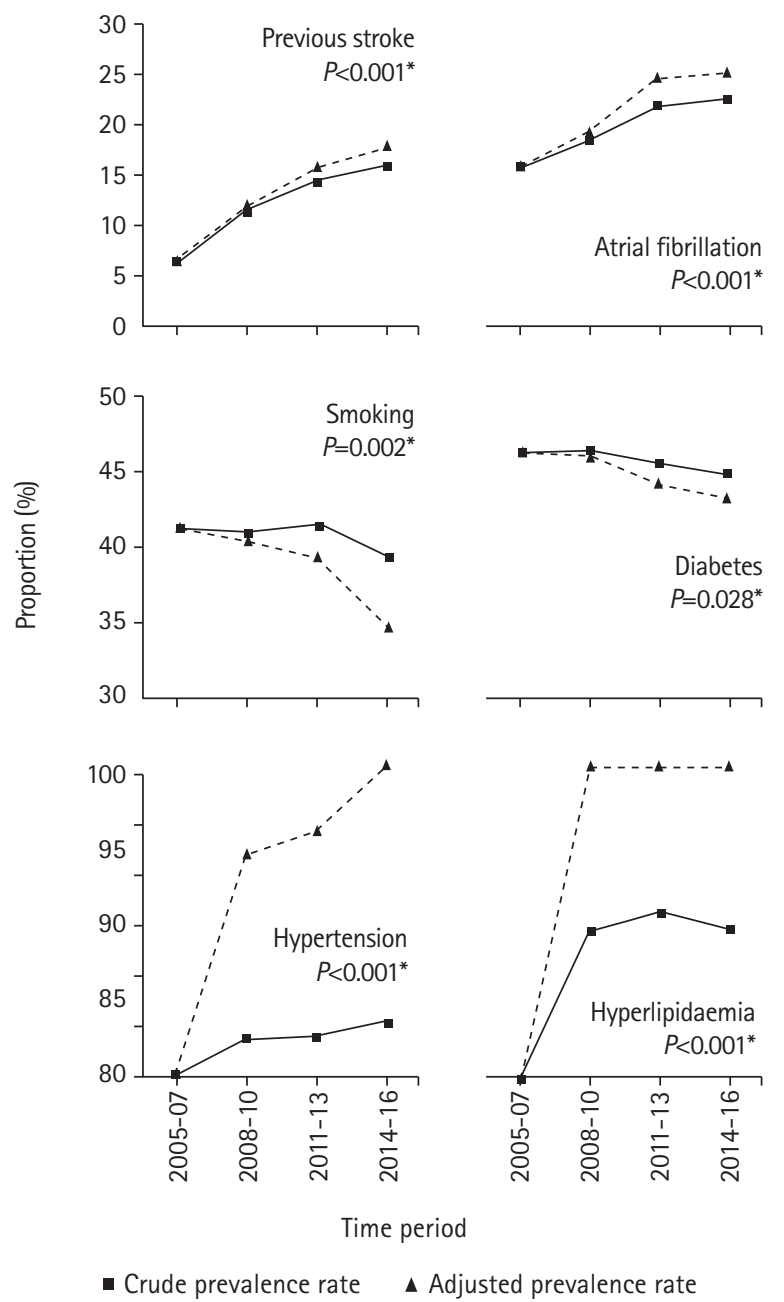

Figure 1. Prevalence of risk factors for acute ischemic stroke over time. *This is shown as a \% of the stroke population. old patients and Malay males, there were risk factors that showed a different trend as compared to the general population of AIS cases. In $<65$-year-old AIS cases, the prevalence of smoking increased from $48.6 \%$ to $51.9 \%$ ( $P<0.001)$. Amongst Malay males with AIS, the prevalence of diabetes increased from 51.7\% to $55.2 \%$ ( $P=0.022$ ).

Our finding of a significant decrease in the overall incidence of AIS across a 12-year period from 2005 to 2016 is consistent with studies done in non-Asian and less heterogeneous populations. ${ }^{2}$ This decrease can be attributed, in part, to reductions in the prevalence of smoking and diabetes, both being common risk factors for AIS, especially in Asia. ${ }^{5}$ These trends are encouraging and worth further exploration to understand how stroke incidence can be further reduced, especially amongst Malays, which has seen an increase in incidence. In contrast to the general population of AIS cases, the increased prevalence of smoking and diabetes amongst younger cases and Malay males respectively may have contributed to the increased incidence of AIS in these subgroups and efforts should be made to tackle these worrying trends. Changes include increasing the legal smoking age from 18 to 21 in $2021,{ }^{6}$ and campaigns aiming to reduce sugar intake during the traditional Malay feasting periods of Ramadan and Hari Raya. ${ }^{7}$ Effects of these changes should be evaluated, ensuring that such trends are reversed.

In the general population of AIS cases, the prevalence of $\mathrm{AF}_{\text {, }}$ hypertension, hyperlipidaemia and previous stroke has increased. More attention should be given to these risk factors, and especially $A F$, given the striking jump in prevalence. The increase in AF prevalence in AIS cases might reflect changes in diagnostic techniques and increased use of technology such as implantable loop recorders and telemetry rather than a true increase in the general population. Furthermore, this trend could also reflect under-utilisation of anticoagulation. ${ }^{8}$ Recent Asia-based studies have reported that patients are often prescribed reduced doses of anticoagulation off-label, ${ }^{9}$ or have lower compliance rates compared to Western populations. ${ }^{10}$ These observations highlight a significant concern that whilst AF prevalence amongst AIS cases in Singapore is increasing, lack of anticoagulant utilisation, under-dosing and compliance issues may lead to increased thromboembolic complications. Further research should be done to ascertain barriers to effective anticoagulation.

We report a significantly higher prevalence of AF amongst Chinese and Malays, as compared to Indians, in our population of AIS cases. There have been studies that suggest reduced prevalence of AF in Indians as compared to Chinese." Larger population-wide studies in Indian communities will help to confirm this and identify possible causative reasons. Future comparative 
studies in these communities using genome-wide association study methodology might also uncover protective heritability factors against AF. ${ }^{12}$ While the strengths of our study lie in its large number of cases and its multi-ethnic composition, it does have limitations. Importantly, the SSR does not classify AIS cases into stroke aetiology subtypes, limiting the analysis of specific reasons behind changes in incidence rates.

In conclusion, while there has been a decreasing incidence of AIS in Singapore in keeping with global trends, this trend is not observed in all subgroups. Our study highlights possible contributing factors, such as smoking in $<65$-year-old AIS cases, and diabetes in male Malay cases. AF has also been shown to be an increasingly important risk factor with clinical implications in different ethnic populations. Observations highlighted in our study could serve as important directions for improved strategies to tackle the growing stroke morbidity.

\section{Supplementary materials}

Supplementary materials related to this article can be found online at https://doi.org/10.5853/jos.2020.00878.

\section{References}

1. O'Donnell MJ, Chin SL, Rangarajan S, Xavier D, Liu L, Zhang $H_{\text {, }}$ et al. Global and regional effects of potentially modifiable risk factors associated with acute stroke in 32 countries (INTERSTROKE): a case-control study. Lancet 2016;388:761-775.

2. Wafa $H A$, Wolfe $C D$, Rudd $A$, Wang Y. Long-term trends in incidence and risk factors for ischaemic stroke subtypes: prospective population study of the South London Stroke Register. PLoS Med 2018;15:e1002669.

3. Ng WK, Goh KJ, George J, Tan CT, Biard A, Donnan GA. A comparative study of stroke subtypes between Asians and Caucasians in two hospital-based stroke registries. Neurol J Southeast Asia 1998;3:19-26.

4. Venketasubramanian N, Chang HM, Chan BP, Young SH, Kong $\mathrm{KH}$, Tang KF, et al. Countrywide stroke incidence, subtypes, management and outcome in a multiethnic Asian population: the Singapore Stroke Registry: methodology. Int J Stroke 2015;10:767-769.
5. Ma RC, Chan JC. Type 2 diabetes in East Asians: similarities and differences with populations in Europe and the United States. Ann N Y Acad Sci 2013;1281:64-91.

6. Minimum legal age for smoking to be raised to 19 on Jan 1. CAN. https://www.channelnewsasia.com/news/singapore/minimum-legal-age-smoking-tobacco-changes-19-jan-11069294. 2018. Accessed July 18, 2020.

7. Neo R. Mosques serve healthier food during iftar this Ramadan. The Straits Times. https://www.straitstimes.com/singapore/mosques-serve-healthier-food-during-iftar-this-ramadan. 2019. Accessed July 18, 2020.

8. Mazurek M, Huisman MV, Rothman KJ, Paquette M, Teutsch $\mathrm{C}$, Diener $\mathrm{HC}$, et al. Regional differences in antithrombotic treatment for atrial fibrillation: insights from the GLORIA-AF phase II registry. Thromb Haemost 2017;117:2376-2388.

9. Lee SR, Lee YS, Park JS, Cha MJ, Kim TH, Park J, et al. Label adherence for non-vitamin $\mathrm{K}$ antagonist oral anticoagulants in a prospective cohort of Asian patients with atrial fibrillation. Yonsei Med J 2019;60:277-284.

10. Tan BY, Leow AS, Teoh HL, Gopinathan A, Yang C, Paliwal PR, et al. High incidence of under-treated atrial fibrillation: perspectives from an Asian Stroke Endovascular Thrombectomy Registry. J Thromb Thrombolysis 2020;49:268-270.

11. Nguyen TN, Hilmer SN, Cumming RG. Review of epidemiology and management of atrial fibrillation in developing countries. Int J Cardiol 2013;167:2412-2420.

12. Roselli C, Chaffin MD, Weng LC, Aeschbacher S, Ahlberg G, Albert $\mathrm{CM}$, et al. Multi-ethnic genome-wide association study for atrial fibrillation. Nat Genet 2018;50:1225-1233.

Correspondence: Leonard Leong-Litt Yeo

Division of Neurology, Department of Medicine, National University Health System, 1E Kent Ridge Road, 119228, Singapore

Tel: $+65-67726532$

Fax: +65-68724101

E-mail: leonardyeoll@gmail.com

https://orcid.org/0000-0002-4249-0402

Received: March 21, 2020

Revised: June 8, 2020

Accepted: June 17, 2020

The authors have no financial conflicts of interest. 
Supplementary Table 1. Demographic data of Singapore residents between 2005 and 2016

\begin{tabular}{|c|c|c|c|c|c|}
\hline & 2005-2007 & 2008-2010 & 2011-2013 & 2014-2016 & $P$ \\
\hline All & $10,576,790(100)$ & $11,148,256(100)$ & $11,452,207(100)$ & $11,706,988(100)$ & \\
\hline \multicolumn{6}{|l|}{ Sex } \\
\hline Male & $5,244,858(49.6)$ & $5,508,857(49.4)$ & $5,639,720(49.2)$ & $5,748,564(49.1)$ & \multirow{2}{*}{0.010} \\
\hline Female & $5,331,932(50.4)$ & $5,639,399(50.6)$ & $5,812,487(50.8)$ & $5,958,424(50.9)$ & \\
\hline \multicolumn{6}{|c|}{ Ethnic group } \\
\hline Chinese & $7,970,078$ (75.4) & $8,286,062(74.3)$ & $8,493,989(74.2)$ & $8,697,559(74.3)$ & 0.228 \\
\hline Male & $3,933,437$ & $4,069,903$ & $4,156,454$ & $4,244,474$ & \\
\hline Female & $4,036,641$ & $4,216,159$ & $4,337,535$ & $4,453,085$ & \\
\hline Malay & $1,457,252(13.8)$ & $1,499,029(13.4)$ & $1,528,993$ (13.4) & $1,563,468$ (13.4) & 0.225 \\
\hline Male & 728,134 & 747,017 & 760,981 & 777,708 & \\
\hline Female & 729,118 & 752,012 & 768,012 & 785,760 & \\
\hline Indian & $907,622(8.6)$ & $1,015,059(9.1)$ & $1,051,728(9.2)$ & $1,064,849(9.1)$ & 0.237 \\
\hline Male & 467,043 & 525,605 & 542,385 & 546,598 & \\
\hline Female & 440,579 & 489,454 & 509,343 & 518,251 & \\
\hline Age (yr) & & & & & 0.036 \\
\hline$<65$ & $9,696,785$ (91.7) & $10,163,947(91.2)$ & $10,316,489(90.1)$ & $10,322,427(88.2)$ & \\
\hline$\geq 65$ & 880,005 (8.3) & $984,309(8.8)$ & 1,135,718 (9.9) & $1,378,886(11.8)$ & \\
\hline
\end{tabular}

Values are presented as number (\%). 